\title{
ZUR ROLLE DER ZWEISPRACHIGKEIT IN AUSGEWÄHLTEN TEXTSORTEN
}

\begin{abstract}
The role of multilingualism in selected text types
The paper deals with the role of multilingualism in selected text types. The reflections on forms of multilingualism and occasions for using multiple languages in a text lead to the creation of a typology that distinguishes between obligatory and optional use of multiple languages in text types. The obligatory multilingualism is constitutive in dictionaries, textbooks and teaching materials. Optional multilingualism, which was examined using the example of German advertising texts and grave inscriptions from Germany and Denmark, reinforces the main function of the text.
\end{abstract}

KEYWORDs: text type linguistics, text type, multilingualism, text function, German

\section{Textsortenlinguistik und ihre Facetten}

Die Textlinguistik als Teildisziplin der Linguistik wird seit etwa 50 Jahren in Forschung und Lehre betrieben und bietet heute ein methodisch sehr differenziertes und inhaltlich erkenntnisreiches Bild. Ihre Forschungsentwicklung kann man zusammenfassen als die Suche nach Zugängen zur Bestimmung der Wesensmerkmale von Texten und zur Charakterisierung einzelner Textphänomene (Heinemann \& Viehweger, 1991, S. 19). Die eher atomistisch ausgerichtete Anfangsphase, in der Ermittlung von transphrastischen Bezügen und folglich von einzelnen Textualitätskriterien in den Vordergrund traten, mündete in die Bemühungen um Bestimmung von Textmustern und Entwicklung des Textsortenbegriffs. Ein nächster Schritt in der Entwicklung der Disziplin waren praxisorientierte Anwendungen, die zu empirischen Analysen von einzelnen Textsorten führten und die eigentliche Untersuchungsrichtung Textsortenforschung/ Textsortenlinguistik im engeren Sinne ins Leben riefen. Die Textsortenlinguistik ging von Anfang an der Frage nach, ,welche zusätzlichen (besonderen) Eigenschaften [...] den konkreten Text als Exemplar einer bestimmten Textsorte" kennzeichnen (Brinker, 1983, S. 127). Schließlich kamen Textlinguisten in den 1980ern zum Schluss, dass sich die Forschung im Bereich der Textsortenlinguistik in drei Strängen bewegt:

Józef Jarosz - Uniwersytet Wrocławski, Wrocław, jozef.jarosz@uni.wroc.pl 
- sie versucht, den Begriff der Textsorte genauer zu definieren,

- sie versucht Klassifikationssysteme und Methoden zur Differenzierung und Relazionierung der verschiedenen Textsorten zu entwickeln,

- sie versucht, einzelne Textsorten zu beschreiben (Lux, 1981, S. 29).

Von ihrer Gültigkeit und langfristigen Aktualität zeugen Postulate, die zwanzig Jahre später von Heinemann (2000) formuliert wurden, dass im Kern des Interessenbereiches der Textsorten-Linguistik folgende Aufgaben mit eingeschlossen werden sollten (Heinemann, 2000, S. 508):

- das prätheoretische Alltagsverständnis von Textsorten explizieren [...],

- den Begriff Textsorte definieren und relevante Konstituenten herausarbeiten,

- $[\ldots]$ das Funktionieren einzelner Textsorten unter unterschiedlichen Konditionen beschreiben,

- die regelhaften (?) Beziehungen zwischen einzelnen Textsorten erfassen und auf dieser Grundlage Typologien von Textsorten aufstellen,

- didaktische Empfehlungen für das Umgehen mit praxisrelevanten Textsorten sowie für die sukzessive systematische Erweiterung und Vertiefung des Textsortenwissens unterschiedlicher Rezipientengruppen entwickeln.

Diesen Richtlinien folgend lieferten Textlinguisten eine Reihe von Textsortenanalysen ab, die im Laufe der Zeit nach verschiedenen Ansätzen und mit einer immer neueren Zielsetzung durchgeführt wurden. Relativ stark - in quantitativer Hinsicht - sind in den Bibliographien (vgl. Schmitt, 1986; Adamzik, 1995'1 Brinker, 2010²) einzelsprachlich synchron angelegte Beschreibungen bestimmter Textsorten vertreten. Einige Textsorten (grundsätzlich Gebrauchstexte) wurden bereits genauer beschrieben - sei es in Aufsätzen, sei es in umfangreicheren Auseinandersetzungen, z. B. Briefsorten (Ermert, 1979), Horoskop (Furthmann, 2006), Gebrauchsanweisung/Bedienungsanleitung (Göpferich, 1995), Kochrezept (Eckkrammer \& Eder, 2000), Rezension (Zillig, 1982), Wetterbericht (Spillner, 1983), Kontaktanzeige (Sommerfeldt, 2002), Todesanzeige (Hosselmann, 2001), Absageschreiben auf Bewerbungen (Szczęk, 2015) und Grabinschrift (Jarosz, 2017). Eingehend wurden 20 Textsorten exemplarisch aus sprachdidaktischer Sicht in der Arbeit von Fandrych \& Thurmair (2011) untersucht (Vorstellungstexte, Reiseführer, Audioguides, Lexikonartikel, Leserbrief, Diskussionsforum, Studienbewertung, Wetterbericht, Horoskop, Bedienungsanleitung, Ordnung, Arztfragebogen, Beschwerde, Anzeigen, Beratungstexte, Tagebuch, Chats, Kondolenztexte, Glückwunschtexte, sprachspielerische Kurzformen). Die Konzeption von Textsorten ${ }^{2}$ erwies sich als besonders fruchtbar für interlinguale Vergleiche (Spillner, 2002, S. 144), die im Rahmen der kontrastiven Textsortenlinguistik betrieben wurden. Als Gründungsdokumente

${ }^{1}$ Die Bibliographie wird online geführt: http://www.unige.ch/lettres/alman/adamzik/akt/aktbibl.html [16.12.2015].

${ }^{2}$ Die Uneinigkeit des Begriffes Textsorte thematisiert Adamzik (1995, S. 11). 
dieses Forschungszweigs - von Anfang an Kontrastive Textologie ${ }^{3}$ genannt - gelten theoretisch und methodologisch fundierte Arbeiten von Hartmann Contrasive Textology (1980) und Spillner Textsorten im Sprachvergleich (1981). Die Liste von bilingualen Textsortenvergleichen wurde bald durch aufwendigere polykonfrontative Analysen bereichert. Die Erweiterung textlinguistischer Forschung durch Einbeziehung von Fachtexten und wissenschaftlichen Texten in das Repertoire der zu untersuchenden Texte führte zur Entstehung eines Forschungsfeldes Fachtextsortenlinguistik, die sich mit den fachsprachlichen Textsorten intralingual und kontrastiv befasst, mit der Annahme, dass auch Fachtexte mehr oder weniger einzelsprachlich spezifischen rhetorischen Traditionen entstammen, durch nationale Traditionen geprägt und kulturell konventionalisiert sind (Spillner, 2002, S. 145). Seit dem sogenannten „cultural turn“ in den 80er Jahren eröffneten kontrastive Textvergleiche eine kulturwissenschaftliche Orientierung in der kontrastiven Textologie ${ }^{4}$, in der man immer öfter der Frage nach der kulturspezifischen und kulturgebundenen Ausprägung von Texten und den interkulturellen Kontrasten von Textsorten nachging. Diesem Ansatz liegt die These zugrunde, dass Textsorten kulturelle Phänomene sind, die auf kulturellen Übereinkünften beruhen (Fix, 2011a, 2011b).

Die anfangs vorherrschende synchrone Ausrichtung der Textsortenlinguistik wurde mit der Zeit überwunden, indem die diachrone Perspektive in das methodische Instrumentarium der Textlinguisten Eingang fand (Steger, 1998, S. 289). Die Beschäftigung mit der Geschichte von Textsorten bzw. Texttypen, die als historische Textsortenlinguistik (vgl. Weidenbusch, 2006a und 2006b, S. 2278) bezeichnet wird, setzt sich zum Ziel, eine Aufgabe ,die in verschiedenen Zeitabschnitten gleichzeitig vorkommenden Texttypen (bezogen auf die kommunikativen Bezugsbereiche, Kommunikationsgruppen und -räume) in synchronen Querschnitten“ (Schenker, 1977, S. 143 f.) zu betrachten, damit unterschiedliche historische Textsortenrepertoires sowie der Wandel der jetzt existenten Textmuster verfolgt und erfasst werden. Immer öfter liegt das Augenmerk auf den durch Medienwandel und Digitalisierung verursachten Veränderungen innerhalb von Textsorten. Diese Forschungslinie befasst sich mit Wandelprozessen der jüngeren Sprachgebrauchsgeschichte (vgl. Beiträge in Hauser \& Kleinberger \& Roth, 2014). Diachron kontrastive Themenstellungen sind - wahrscheinlich wegen des Aufwands in wenigen Arbeiten in Angriff genommen worden ${ }^{5}$. Der neueste Trend im Bereich der

${ }^{3}$ Der Terminus Textologie war kurze Zeit in synonymischer Konkurrenz zu der von H. Weinrich geprägten Bezeichnung Textlinguistik. Etwas später wurde der Begriff Textologie in Verbindung mit dem Adjektiv kontrastiv zur Bezeichnung von vergleichenden Untersuchungen von Textsorten wieder aufgenommen (Pöckl, 2010, S. 729).

${ }^{4}$ Ausführlicher darüber s. Adamzik (im Druck) und Fix (2011a, 2011b).

${ }^{5}$ Linguistische Zeitschriftenartikel aus Deutschland und Finnland waren Untersuchungsgegenstand in der monographischen Darstellung von Szurawitzki (2011), deutsche und chinesische wissenschaftliche Rezensionen kontrastierte Ziting (2013). Deutsche und finnische Kontaktanzeigen im 20. Jahrhundert wur- 
Textsortenlinguistik liegt außerhalb einer Textsorte und umfasst die Untersuchung von Textsortennetzen, in der betreffende Textsorte in einen größeren Kommunikations- und Funktionskontext gestellt wird, wodurch der komplexe kommunikative Umgang mit gesellschaftlichen Ereignissen und Phänomenen innersprachlich sowie interkulturell beobachtet werden kann (z. B. Jarosz, 2015). Als Zukunftsmusik ist schließlich wohl die Etablierung von Eurotextologie in konsequenter Auslegung aufzufassen, die als „flächendeckende Beschreibung einer repräsentativen Anzahl von Textsorten der europäischen Sprach- und Kulturräume" verstanden werden soll (vgl. Pöckl, 2010, S. 729-730).

Diese in äußerster Kürze dargestellte Entwicklung der Textsortenlinguistik zeigt eine Menge von Ansätzen, Fragestellungen, Zielsetzungen und Erkenntnisinteressen in den bisherigen Aktivitäten von Textlinguisten des deutschsprachigen Raumes und erschöpft nicht alle Untersuchungsdomänen, die in der Forschungslandschaft der letzten 40 Jahre Fuß fassten. Überraschenderweise wurde dem Phänomen der Zwei- oder Mehrsprachigkeit ${ }^{6}$, die als Textualitätsmerkmal betrachtet wird, in konkreten Textsorten mit Fokus auf ihre Formen und Funktionen bisher kaum Aufmerksamkeit geschenkt.

In dem vorliegenden Beitrag, der in knapper Form den Gebrauch zweier bzw. mehrerer Sprachen in unterschiedlichen Textsorten aufgreift und an einigen Beispielen näher betrachtet, wird ein Versuch unternommen, diese Lücke zu schließen. Anhand der extralingualen Faktoren wird der Status von Mehrsprachigkeit aus der Perspektive des Rezipienten näher beleuchtet und es wird eine funktionale Typologie mit dem Erkenntnisziel erstellt, die Funktion der Zweisprachigkeit hinsichtlich der Textkonstitution und -funktion zu ermitteln. Dabei wird von der Annahme von Hartmann, eines der Gründerväter der Textlinguistik, in seinem berühmten Diktum ausgegangen, dass Sprache nur als Text vorkommt, der als Basiseinheit des Sprachgebrauchs als originäres sprachliches Zeichen gilt (Hartmann, 1971, S. 10 f.).

Der Begriff Mehrsprachigkeit dient in der Regel zur Bezeichnung einer Fähigkeit von Menschen, in mehr als einer Sprache zu kommunizieren oder ist mit der Anwendung mehrerer Sprachen in einem Gebiet gleichzusetzen. Wird der Begriff auf Textsorten übertragen, ist er als Gebrauch von mehreren (mehr oder weniger) gleichberechtigten Sprachen in einem Text zu verstehen.

\section{Textsorten und Mehrsprachigkeit}

Verbale Kommunikation in einer ethnisch und sprachlich homogenen Kommunikationsgemeinschaft verläuft normalerweise durch Produktion und Rezeption von monolingualen Texten, sei es in gesprochener, sei es in geschriebener Form. Selbstver-

den von Rinnekangas (2013) gegenübergestellt. Ausgewählte Fernsehnachrichten-Textsorten analysierte diachron wie auch sprach- und kulturvergleichend Luginbühl (2014).

${ }^{6}$ Aus sprachökonomischen Gründen wird in den folgenden Ausführungen über Zweisprachigkeit statt Zwei-/Mehrsprachigkeit die Rede sein. 
ständlich gilt das Modell in einer multilingualen Gesellschaft mit dem unterschiedlichen bzw. gleichwertigen Status einzelner Sprachen als nicht ausreichend. Eine zwei- oder mehrsprachige Kommunikation im offiziellen Sprachgebrauch resultiert gewöhnlich aus Respekt für Vertreter beider Sprachen und/oder Nationalitäten, sowie aus pro-egalitarischer Absicht, im Idealfall alle Kommunikationsteilnehmer gleichwertig zu behandeln (vgl. Jarosz, 2013, S. 117)7. Diese Umstände scheinen ein natürlicher Anlass zur Entstehung zweisprachiger Texte zu sein. Hinter dieser allgemeinformulierten Diglossie von Textsorten verbergen sich eine Anzahl von Beweggründen und Argumenten für den Gebrauch mehrerer Sprachen bei der Textproduktion und einige Paradigmen bei der Textrezeption. Bei der Untersuchung von Zweisprachigkeit in Textsorten sind m. E. je nach dem Verhältnis zwischen einzelnen Textexemplaren und Gebrauch mehrerer Sprachen - wenigstens zwei Konstellationen zu erwarten:

- ein komplettes Textexemplar liegt in einigen Sprachversionen vor,

- ein Textexemplar besteht aus Komponenten, die in verschiedenen Sprachen abgefasst wurden.

Zur expliziten Differenzierung beider Situationen wird in der vorliegenden Untersuchung von mir eine Terminologie eingeführt, die das Phänomen aus der Perspektive der inneren Textstruktur heraus betrachtet: Die erste Variante der Zweisprachigkeit (a) wird als ,textexterne“ Zweisprachigkeit bezeichnet, d. h., jedes Textexemplar ist einsprachig und die einzelnen Textkomponenten werden von dem Gebrauch mehrerer Sprachen nicht betroffen; ,textinterne“ Zweisprachigkeit bezieht sich dagegen auf den Gebrauch mehrerer Sprachen in den Textgrenzen eines Textexemplars (b) ${ }^{8}$, d. h., einzelne Textbausteine wurden in zwei verschiedenen Sprachen abgefasst. Hier drängt sich die Frage nach dem quantitativen Verhältnis beider/mehrerer Sprachen zueinander in einem Textauftritt auf. Damit nicht jedes Textexemplar, in dem einige Sprachen nebeneinander in verschiedenen Funktionen koexistieren ${ }^{9}$, als zweisprachiger Text betrachtet wird, wird hier folgendes Kriterium angenommen: Als zweisprachiger Text gelten diejenigen kommunikativen Auftritte (d. h. Texte), in denen einzelne Textkomponenten, das heißt Teiltexte oder Textbausteine, in unterschiedlichen Sprachen verfasst sind. Erst dieser Sachverhalt gewährleistet eine relative Gleichberechtigung der gebrauchten Sprachen als Sprachen der Narration/Beschreibung oder Sprachen der Textbildung.

${ }^{7}$ Bilingualität und Bikulturalität sind beim Individuum in der Regel nicht gleichmäßig verteilt. Auf das Problem der Gleichwertigkeit von Sprachen und Textsorten in multilingualen Gebieten verweist Hofeneder (2013, S. 46) am Beispiel der Ukraine: Russisch war bei allen Textsorten vertreten und besaß folglich ein Informationsmonopol, während das Ukrainische auf einige wenige Textsorten beschränkt blieb.

${ }^{8}$ Die formale Übereinstimmung der Bezeichnungen textintern und textextern mit den Kriterien zur Zuordnung von Texten zu Textsorten (Textsortenklassifikation) ist zufällig.

${ }^{9}$ Wie es z. B. in unzähligen linguistischen Texten mit zahlreichen fremdsprachlichen Zitaten bzw. Beispielen und lateinischen Ausdrücken und Einschüben, die teilweise als stilistische Mittel oder fachliche Bezeichnungen dienen (wie z. B. pars pro toto, par excellence, in absentia, casus rectus, casus obliquus usw.), der Fall ist. 
Alle anderen Konstellationen gelten als monolinguale Texte mit fremdsprachlichen Beispielen/Zitaten bzw. stilistisch begründeten Einschüben. Die Gliederung dieser Art ergibt eine mehr oder weniger abgeschlossene Liste von Textsorten, die in beide Situationen verwickelt werden können.

\section{3. „Textexterne“ Zweisprachigkeit von Textsorten}

Diese Erscheinungsform des diskutierten Phänomens gilt wohl als allgemeingültige und meist verbreitete Auffassung von Zweisprachigkeit. Die textexterne Mehrsprachigkeit setzt das Vorhandensein des gleichen bzw. fast gleichen Textinhalts in zwei/mehreren Sprachen voraus. Dabei stehen alle Textexemplare in verschiedenen Sprachfassungen in der Relation der denotativen, konnotativen bzw. funktionalen Äquivalenz zueinander und die jeweiligen Sprachvarianten gelten als zielsprachliche Entsprechungen, die infolge der sprachlichen Operation an einem Ausgangstext (Übersetzung) entstanden sind und Kommunikation in einer tatsächlich zweisprachigen oder potenziell zweisprachigen Gesellschaft sichern sollen. Als selbstverständliche Beispiele seien hier Informationstafeln, Eigennamen (von Ämtern, Institutionen, Straßen usw.), Fahrpläne, Überschriften, Broschüren, Zeitschriften und Zeitungen (mit zahlreichen Textsorten) zu nennen ${ }^{10}$. Auf diesem Prinzip bauen weitere Beispiele auf, die, abgesehen von der Situation der natürlichen Zweisprachigkeit, in zwei Sprachen verfasst wurden. Es handelt sich um Reisedokumente (Reisepass, Fahrkarten, Versicherungspolicen), Formulare und Anträge, Produktbeschriftungen, Broschüren mit Gebrauchsanweisungen und Bedienungsanleitungen, Packungsbeilagen, Zusammensetzungen von Produkten, die das gleiche Textexemplar in mehreren Sprachvarianten enthalten ${ }^{11}$. Die genannten Kompilationen sind nur oberflächlich betrachtet mehrsprachig, weil sie sich im Grunde genommen an einsprachige Rezipienten wenden und jeweils einsprachig rezipiert werden, denn die übrigen Sprachversionen bleiben ohne Einfluss auf die Textkonstitution, Textfunktion, Gesamtbotschaft sowie Rezeption. Sie haben als autonome Entitäten ihre Geltung und befinden sich nur aus praktischen Gründen auf einem Träger (z. B. Reisepass, Fahrkarten), auf einer Produktverpackung oder in einer Informationsbroschüre eines Produkts, das in mehreren Ländern verkauft wird. Zwischen den Texten in mehreren Sprachen besteht das gleiche Verhältnis und sie entstanden infolge einer identischen Operation am Text (d. h. Textübersetzung in

${ }^{10}$ Zweisprachige Zeitungen haben in multilingualen und multikulturellen Gebieten eine lange Tradition. Es sei hier beispielsweise der Lodzer Anzeiger (Lódzkie Ogłoszenia) genannt, die erste zweisprachige Zeitung in Łódź, die 1863 gegründet wurde (Weigt, 2013, S. 353).

${ }^{11}$ Unter literarischen Texten sind u. a. mehrsprachige Gedichtbände oder Sammlungen von Sprichwörtern zu nennen. Die letzteren gehen bereits im 16. Jahrhundert in die Kulturgeschichte der einzelnen Sprachgemeinschaften ein, wie z. B. die erste lateinisch-dänische Kompilation von Sprichwörtern 1506 (Jarosz, 2011, S. 466). 
Anlehnung an alle in der Übersetzungswissenschaft geltenden Regeln). Der Gebrauch mehrerer Sprachen ermöglicht die Kommunikation zwischen dem Textproduzenten und den monolingualen Textrezipienten aus verschiedenen kommunikativen Gemeinschaften, leistet aber keinen Beitrag zur Textbedeutung und wird kaum beachtet oder gar nicht von monolingualen Rezipienten wahrgenommen.

\section{4. „Textinterne“ Zweisprachigkeit}

Bei der textinternen Mehrsprachigkeit liegen Textexemplare vor, deren einzelne Textsequenzen (Teiltexte, Textkomponenten) in zwei/mehreren Sprachen verfasst wurden. Textexemplare aus dieser Gruppe stellen somit eine Sprachmischung dar und die Mehrsprachigkeit manifestiert sich innerhalb von Delimitationssignalen, d. h. zwischen Initiatoren und Terminatoren (Werlich, 1975, S. 17) eines Textes dadurch, dass einzelne Textteile in unterschiedlichen Sprachen abgefasst sind und nebeneinander stehen oder nacheinander folgen und eine thematische Kontinuität sowie einen übergreifenden Bedeutungszusammenhang bilden. Bei der Bestimmung des Status der Zweisprachigkeit (begriffen als Textualitätsmerkmal) in Textsorten dieser Art ist die Frage nach den Ursachen der Anwesenheit mehrerer Sprachen in einem Text ein ausschlaggebendes Kriterium. Danach unterscheidet man Texte, die obligatorisch, d. h. unabhängig von den Absichten und Intentionen des Textproduzenten, mehrsprachig sind, und solche, in denen die Verwendung von mehreren Sprachen fakultativ ist und die Entscheidung über den Sprachgebrauch in diesem Bereich dem Textproduzenten überlassen wird.

\subsection{Obligatorische Zweisprachigkeit (Wörterbücher, Lehrbücher, Lehrmaterialien)}

Ein reibungsloses Nebeneinander zweier Sprachen ist eine Selbstständigkeit in einer Reihe von Textsorten im didaktischen Bereich. Gemeint sind hier in erster Linie bi- und multilinguale Wörterbücher (darunter Wörterbücher mit alphabetischer Ordnung, sowie Thesauri, Bild-, Fachwörterbücher u. a.) und Sprachlehrbücher (u. a. zweisprachige Sprachführer und Lehrbücher für Selbstlerner) sowie Materialien zur Ausbildung von Übersetzern und Dolmetschern (z. B. Publikationen mit Paralleltexten). Das Lehrbuch gehört neben der Schulgrammatik und Lektion (als Teil eines Lehrbuches zur Didaktik einer Fremdsprache) zu den assertiven Textsorten, die ein als gesichert geltendes Wissen darstellen (Rolf, 1993, S. 197). Von der Liste der betreffenden Textsorten sind monolinguale Grammatiklehrbücher auszuschließen ${ }^{12}$. Bilingualität ist in diesen Textsorten

\footnotetext{
${ }^{12}$ Sie werden in der Regel in einer Sprache verfasst und reichlich mit zielsprachlichen Beispielen belegt (z. B. Łuszczyk \& Szulc \& Wawrzyniak, 1988). Zu seltenen Publikationen gehören zweisprachige Lehrbücher sensu stricto, in denen jede Doppelseite aus Erläuterungen in einer Fremdsprache (auf der
} 
ein unentbehrliches Aufbauprinzip, das ihre Grundfunktion und Grundbestimmung realisierbar macht, d. h. die Befriedigung von Informationsbedürfnissen, die auf Gewährleistung von Kommunikation abzielen. Die Benutzung von mehrsprachigen Wörterbüchern/Lehrbüchern dient dem Ziel (ohne auf die Einzelheiten und funktionale Differenzierung der genannten Textsorten einzugehen), die Sprachkompetenz im Bereich einer Fremdsprache zu kontrollieren und bei ihrem Erwerb behilflich zu sein, besonders bei der Rezeption oder Produktion eines fremdsprachlichen Textes. Die Textsorten mit ihrem zweisprachigen Konzept vermitteln den Lernenden Wissen über Sprache und Kultur sowie Sprachfertigkeiten, bzw. verlangen ihnen diese ab. Im Einzelnen geht es in lexikographischen Kompendien und Lehrbüchern u. a. um die Wiedergabe der denotativen und teilweise konnotativen Bedeutung einzelner Lexeme, Phraseologismen, Sprichwörter und schließlich die Sinnerschließung des Ausgangstextes, was die Bestimmung von zwischensprachlichen Relationen auf verschiedenen Sprachebenen zur Folge hat, mit anderen Worten: die Herausbildung und Förderung von Kompetenz im Bereich der Bilingualität. Der Wissens- und Kompetenztransfer erfolgt grundsätzlich mithilfe metasprachlicher Funktion, die in den Textsorten dieser Art eine Schlüsselrolle spielt. Als Zielgruppe gilt hier zweisprachiges bzw. zweisprachiges Publikum in spe. Da die Erfüllung der bereits genannten Funktionen ohne simultane Kookkurrenz zweier Sprachen undenkbar ist, gilt die Zweisprachigkeit als ein konstitutives Merkmal und somit steht ihr die Funktion der Textkonstitution zu.

\subsection{Fakultative Zweisprachigkeit. Mehrsprachige Sammel- und Gedichtbände}

Hier handelt es sich z. B. um Sammelbände, Anthologien und Sammlungen von Texten, die von einem Autor/einigen Autoren in mehreren Sprachen (wissenschaftliche, populärwissenschaftliche Sammelbände, zweisprachige Gedichtbände) verfasst wurden. Bei der Textsorte Sammelband hat die Mehrsprachigkeit keinen Einfluss auf den kommunikativen Wert der Texte. Es besteht keine direkte Beziehung (z. B. Äquivalenz) zwischen Text A in der Sprache A und Text B in der Sprache B. Hätte man alle Texte in einer Sprache verfasst, wäre diese Tatsache ohne Bedeutung gewesen. Die Sprachen, in denen einzelne Beiträge verfasst wurden, charakterisieren vor allem den Absender und geben Auskunft über seine Muttersprache bzw. seine Fremdsprachenkenntnisse und sind keine weitere Erkenntnisquelle in dem Sinne, der das Sujet der vorliegenden Ausführungen ist.

Separat ist m. E. die interne Zweisprachigkeit von Gedichtbänden zu betrachten, in denen einzelne Gedichte in zwei Sprachfassungen in einem starken intertextuellen Bezug (Ausgangstext - Zieltext) stehen. Ob die zweisprachige Version von Gedichten 
einen Einfluss auf die Rezeption eines Textes ausübt, hängt von den Textempfängern ab. Hier ist nämlich die Sprachkompetenz des Rezipienten ausschlaggebend und muss in den Rezeptionsprozess einbezogen werden. Bei monolingualen Rezipienten spielt der Text in der Originalform keine Rolle. Aufgrund der Spezifik der literarischen Übersetzung kann der Originaltext bei bilingualen Rezipienten als eine zusätzliche Erkenntnisquelle gelten, die die Einzelheiten der Übersetzung vervollständigt ${ }^{13}$. Dies kann als Mehrwert bilingualer Ausgaben von literarischen Texten betrachtet werden, was die folgenden Beispiele illustrieren:

1) Na przedzie xiążę,

Najpochlebniej niebrzuchaty,

Przy xiążęciu xiężna pani,

Cudnie młoda, młodziusieńka [...].

2) Ledwie wnijdę, słów kilka przemówię z nią samą, Jużci dzwonek przeraża, wpada galonowy [...].
Allen voran der Fürst, am allenschmeichelhaftesten unbeleibt, neben dem Fürsten Frau Fürstin, wunderbar jung, allerjüngst $[\ldots]{ }^{14}$

Ich komm und sage, allein mit ihr, einen Satz, Da schrillt die Glocke, der Diener dringt ein [...]. ${ }^{15}$

In den beiden Belegen ist der translatorische Verlust bei der Wiedergabe der veralteten Schriftweise, die als Stilmittel dient (Beispiel 1), und der authentischen Archaismen (Beispiel 2) auffällig. Die Feststellung dessen Existenz setzt allerdings angemessene sprachliche Kompetenz des Rezipienten voraus. Erst diese ermöglicht einen Einblick in die Technik des Übersetzens und folglich einen (privaten oder professionellen) Übersetzungsvergleich und die Ermittlung zwischensprachlicher Kontraste in einem poetischen Text.

\subsection{Fakultative Mehrsprachigkeit. Zweisprachigkeit in Werbetexten}

Im Falle von Werbeanzeigen, deren Umfang eher gering ist und sich auf einige Sätze bzw. satzäquivalente Einheiten beschränkt, ist eine breite Palette von Beispielen nachzuweisen, in denen die Anwesenheit der fremdsprachlichen Elemente Verwendung findet. Dabei erstreckt sich ihr Anteil von einzelnen Lexemen, über Phrasen und Phraseologismen bis hin zu vollständigen Sätzen (Slogans) oder sie beherrschen gar die ganze verbale Komponente eines Werbetextes. Somit kann man von fremdsprach-

${ }^{13}$ Gemeint sind hier Textstellen, die wegen struktureller Unterschiede zwischen den Sprachen auf verschiedenen Ebenen, mit Verlust übertragen werden müssen. Es geht hier vor allem um Beispiele für Nulläquivalenz, Unübersetzbarkeit, Veränderungen im stilistischen Bereich (stilistische Absenkung oder Erhebung) und im semantischen Bereich (Gebrauch von Paraphrase, Auslassung, funktionalen Entsprechungen u. a.). Die bilingualen Rezipienten können die Verluste durch die Lektüre des Originaltextes nachvollziehen.

${ }^{14}$ Das angeführte Fragment wurde dem Gedicht von Wisława Szymborska Miniatura średniowieczna, übers. von Karl Dedecius (Mittelalterliche Miniatur) (Dedecius, 1982, S. 224-225) entnommen.

${ }^{15}$ Das Fragment stammt aus dem Gedicht von Adam Mickiewicz Do D. D., übersetzt von B.K. Tragelehn (An D. D.) aus der Sammlung von Mickiewicz (1979, S. 78-79). 
lichen Einschüben mit explizit vorliegender Dominanz der einen Sprache oder über Zweisprachigkeit eines bestimmten Textes sprechen.

Werbeanzeigen, genauso wie andere Erscheinungsformen der kommunikativen Praxis im Bereich der Werbung, repräsentieren nach textlinguistischen Kriterien der Textsortenklassifizierung direktive, nicht-bindende Textsorten, deren Hauptfunktion darin besteht, Personen zu einer Aktion zu bewegen, deren Ausführung sowohl im Interesse des jeweiligen Textproduzenten als auch des Adressaten liegt (Rolf, 1993, S. 250).

Werbung wird die geplante, öffentliche Übermittlung von Nachrichten dann genannt, wenn die Nachricht das Urteilen und/oder Handeln bestimmter Gruppen beeinflussen und damit einer Güter, Leistungen oder Ideen produzierenden oder absetzenden Gruppe oder Institution (vergrößernd, erhaltend oder bei der Verwirklichung ihrer Aufgaben) dienen soll. (Hoffmann, 1981, S. 10)

Da das Phänomen der fremdsprachigen (vor allem anglo-amerikanischen) Einflüsse auf die Werbesprache in Deutschland seit Jahrzehnten und zwar mit zunehmender Tendenz zu beobachten ist ${ }^{16}$, wurde es Untersuchungsgegenstand zahlreicher Studien (u. a. Bohmann, 1996; Schütte, 1996; Kupper, 2007) ${ }^{17}$. Im Folgenden wird nur auf die Funktion der Mehrsprachigkeit in der diskutierten Textsorte, nicht dagegen auf die detaillierte Darstellung der Erscheinungsformen oder Kreativität der Textproduzenten eingegangen. Auch andere Aspekte werden lediglich angedeutet.

Funktionen von Texten gehen eher vom Sender aus, die Wirkung ist dagegen empfängerbezogen. Es ist schwer beides klar zu trennen (Elsen, 2008, S. 91). Anzumerken bleibt, dass unterschiedliche Textbausteine in Werbetexten mit unterschiedlicher Häufigkeit von Gebrauch fremdsprachlicher Elemente betroffen sind. Das Gleiche gilt für die Präsenz von Fremdsprachen in der deutschen Werbung. Lässt sich die Hegemonie des Englischen hier nicht verbergen, hat das Englische bei manchen Produktgruppen kein Monopol, denn die Wahl der Fremdsprache in Einzelfällen ist doch von der beworbenen Produktgruppe abhängig ${ }^{18}$. Überdurchschnittlich oft kommen englischsprachige Wendungen in Slogans von Firmen vor. Diese kurzen Äußerungen, die als

${ }^{16} \mathrm{Zu}$ unterscheiden ist zwischen dem Gebrauch authentischer englischer Lexik und Phraseologie und dem Gebrauch von Pseudo-Fachwörtern oder hybriden Formen (Scheinentlehnungen), die dem Englischen ähneln und kaum im Gebrauch in den englischsprachigen Ländern sind (z. B. Handy), oder Wortbildungen mit Elementen aus zwei verschiedenen Sprachen (z. B. Air-Schaum-System).

${ }^{17}$ Vgl. u. a. die breit angelegte Studie von Schütte (1996), in der die Verwendung von Anglizismen in der deutschen Zeitschriftenwerbung diachronisch von 1951 bis 1991 untersucht wurde. Schütte zufolge ist statistisch erwiesen, dass Anglizismen eher in der Werbung für Mode, Technik, Reisen, Kosmetik, Alkoholika und Zigaretten als in der tendenziell anglizismenärmeren Werbung für Dienstleistungen, Arzneimittel und alltägliche Konsumgüter zu erwarten sind.

${ }^{18} \mathrm{Im}$ Kosmetikbereich wird oft die Verwendung des Französischen bevorzugt, das Assoziationen von Eleganz, Luxus und Schönheit mit sich bringt. Italienische Slogans stehen oft mit Produkten der Nahrungsindustrie bzw. kulinarischen Spezialitäten aus Italien im Einklang. In der Werbung für einheimische Produkte kann der Gebrauch von fremdsprachlichen Komponenten als ein Fauxpas betrachtet werden. Zur Entwicklung der Sprachwahl nach Branche s. Andropolous et al. (2004, Kap. 3.2). 
Werbesprüche, also relativ autonome Textkomponenten einer Werbeanzeige gelten, sind meist komplett in Englisch abgefasst, z. B. Come in and find out (Douglas). Mit dieser Textkomponente kontrastieren andere Textbausteine von Werbeanzeigen wie Fließtexte, die überwiegend im verständlichen Deutsch geschrieben werden, weil diese Textsequenzen für sachliche und zuverlässige Information (auch wenn in knapper Form verfasst) zuständig sind und somit das Postulat der Verständlichkeit in möglichst hohem Grad realisieren müssen. Englische Textsequenzen treten also vornehmlich in exponierten Textbausteinen (Slogans, Firmennamen) auf, und übernehmen damit die Rolle, als Erstes die Aufmerksamkeit der Rezipienten zu wecken. Dies erfolgt durch einen Überraschungseffekt, der manchmal selbst durch den Einsatz fremdsprachlicher Elemente erreicht und durch sprachspielerische Einbettung oder Sprachmischung gesteigert wird. Damit ist die Liste von Funktionen, die fremdsprachlichen Textkomponenten zukommt, nicht erschöpft ${ }^{19}$. Oft verbindet man mit dem englischen im Gegensatz zum deutschen Begriff positive Konnotationen: Boss klingt moderner und attraktiver als Vorgesetzter. Englische Fachwörter oder komplexere Formen, die in der Computerwerbung verwendet werden, können technischen Fortschritt suggerieren. In der Zigarettenwerbung vermittelt das Englische die Vorstellung von Freiheit, Weite, dem American way of life (wie es bei der Marlboro- und Camel-Werbung mit der Darstellung von Cowboys in der Wildnis seit Jahrzehnten der Fall ist). In der Kosmetik- und Pflegemittelwerbung wird häufig Internationalität, Individualität, Weltoffenheit, Jugend und Lebensfreude, Fortschritt und Qualitat über das englische Wortgut transportiert (Elsen, 2008, S. 91). Außerdem soll nicht unerwähnt bleiben, dass Anglizismen andere positive Assoziationen auslösen können: „Meist klingt Englisch fachlich, gehoben oder geheimnisvoll, exklusiv, modern, im Gegensatz zu deutschen Formulierungen, die eher mit gewöhnlich, langweilig, bieder, teilweise auch neutral, nüchtern oder sogar lächerlich in Verbindung gebracht werden" (Elsen, 2008, S. 92). In Werbeslogans dient das Englische in erster Linie dem Zweck, Modernität, Dynamik, Jugendlichkeit und Internationalität zu manifestieren oder wenigstens vorzutäuschen. Die Verwendung fremden Wortguts kann darüber hinaus in Werbetexten ein Lokalkolorit verleihen. Als ein weiterer Aspekt ist die Sprachökonomie zu erwähnen. Viele englische Lexeme, sowie Slogans sind von ihrer Silbenzahl her oft kürzer als ihre deutschen Entsprechungen, z. B. after shave nach der Rasur, fit - in bester Verfassung, set-Garnitur, Satz u. a., was sie besonders für knappe Formulierungen in Werbe-Textsorten prädestiniert (vgl. Bohmann, 1996, S. 191 ff.). Die Wörter sind ökonomischer und haben einen frischen Hauch, außerdem sind sie - wegen steigender Englischkenntnisse - in der Regel verständlich und in die deutsche Sprache quasi integriert, weil sie immer öfter in Werbetexten und in

${ }^{19}$ Es sei am Rande angemerkt, dass der Gebrauch des Englischen in Namen und Beschreibung der Bestimmung von Produkten auch negative Folgen wegen des geringeren Informationswertes haben kann, weil der Text für manche Rezipienten nicht verständlich ist, wodurch die genaue Wirkweise von Produkten nicht klar ist. Dies kann manche Kunden verunsichern und auf einige sogar abschreckend wirken (vgl. Elsen, 2008, S. 92). 
den Medien verwendet und rezipiert werden ${ }^{20}$. In der Regel handelt es sich um eine semantische Aufwertung, die dadurch erfolgt, dass Anglizismen als Wert- und Emotionsträger auftreten (vgl. Bohmann, 1996, S. 185). Kurz gesagt: Das Fremde (in der Sprache) ist attraktiv, verspricht ein gelungenes Einkaufserlebnis und zieht an. Als Beispiele für einige geschickte Werbeslogans seien hier genannt ${ }^{21}$ : There's no better way to fly (Lufthansa), Come in and find out (Douglas), Welcome to the Beck's experiment (Beck's), Ford - feel the difference (Ford), Drive Alive (Mitsubishi).

Die bisherigen Beobachtungen führen zur Schlussfolgerung, dass dem Gebrauch von Anglizismen vorwiegend wirkungsintentionale Motive zu Grunde liegen. Die Präsenz und die stilistische Funktion des Englischen sollen dabei die Werbewirkung steigern, d. h., sie dienen als weitere Mittel zur Steuerung der Aufmerksamkeit, Denkund Verhaltensweise der Rezipienten. Man kann somit den fremdsprachigen Elementen in Werbeanzeigen die Hauptfunktion der Textsorte zuschreiben: Appellfunktion und persuasive Wirkung.

\subsection{Fakultative Mehrsprachigkeit. Grabinschriften}

Als Beispiel für eine andere Textsorte, die von ihrer Natur her monolingual ist, deren Zweisprachigkeit außersprachliche Faktoren bedingen, ist die Grabinschrift ${ }^{22}$. Seit ihrem Ursprung erfüllte die Grabinschrift vor allem die Funktion, die Stätte der Beerdigung zu markieren, ihr einen individuellen Charakter zu verleihen, die Person des Verstorbenen zu identifizieren und zu charakterisieren, und eine Nebenfunktion: die Umwelt über das Ableben einer Person zu informieren. Grabinschriften bekunden, trotz ihres geringen Umfangs, was den Hinterbliebenen und öfters auch den Toten wichtig war, und sie bewahren es für die kommenden Generationen (Guthke, 2006, S. 15).

Nach der Taxonomie von Rolf(1993, S. 216) vertritt die Grabinschrift, ,inzitierende Textsorten“ (im Bereich der sepulkralen Assertiva, vgl. Jarosz, 2015, S. 29-30), deren Funktion darin besteht, zum Denken anzuregen, bestimmte mentale Prozesse in Gang zu setzen, z. B. solche, die eine Entscheidung finden oder bestimmte Erfahrungen verarbeiten helfen. Diese Textsorten erzielen nicht die Hervorbringung einer Handlung (wie es bei den direktiven Textsorten der Fall ist), sondern bestimmte mentale Prozesse. Ob etwas aus diesen resultiert, bleibt kaum von Interesse (vgl. Rolf, 1993, S. 216).

${ }^{20}$ Desweiteren können internationale Konzerne mit demselben englischen Werbeslogan Werbekampagnen weltweit veranstalten.

${ }^{21}$ Alle Beispiele wurden der Website http://www.sueddeutsche.de/leben/englische-werbeslogans-komm-rein-und-finde-raus-1.141830-7 entnommen [9.12.2015].

${ }^{22}$ Die mehrsprachige Außenwerbung sowie Grabinschriften zählen zur visuellen, d. h. sichtbaren Mehrsprachigkeit, weil sie an öffentlichen Stellen zugänglich sind (vgl. Ziegler, 2013, S. 299). 
Die Koexistenz von zwei Sprachen in dieser Textsorte wird am Beispiel von Textbelegen von deutschen und dänischen Friedhöfen erörtert. Ausgezeichnetes Material zur Veranschaulichung der Zweisprachigkeit in älteren Grabinschriften bieten jüdische deutsch-hebräische Grabinschriften ${ }^{23}$.

(3)

\begin{tabular}{|c|c|}
\hline Hier ist geborgen & $\begin{array}{l}\text { Qפ"ט } \\
\text { des } \\
\text { liebreichen Gatten und } \\
\text { treubesorgten Vaters, } \\
\text { Herrn } \\
\text { Liebmann Steinhardt, } \\
\text { geb. zu Dittigheim in Baden } \\
\text { am 13. Novbr. 1827 } \\
\text { gest. dahier am 22. Juni } 1889 \\
\text { (23. Siwan 5649) }\end{array}$ \\
\hline $\begin{array}{l}\text { >der lautere und aufrechte Mann`, der geehrte Elieser, Sohn } \\
\text { des geehrten Josef SeGaL, und sein Andenken zum Segen, } \\
\text { er kehrte zurück in seine Welt am Tag des heiligen Schabbat, } \\
\text { 23. Sivan } 649 \text { der kleinen Zählung. }\end{array}$ & $\begin{array}{l}\text { האיש תם וישר כ' אליעזר בן כ' יוסף סג"ל וז"ל לעולמו ביום ש"ק כ"ג סיון תרמ"ט לפ"ק Er wandelte untadelig, übte Recht, } \\
\text { und redete vom Herzen Wahrheit.“ } \\
\text { (Ps. 15,2.) }\end{array}$ \\
\hline Seine Seele sei eingebunden in das Bündel des Lebens & \\
\hline
\end{tabular}

Diese Grabinschrift (3) ist ein Beispiel für einen zweisprachigen sepulkralen Text, in dem beide Sprachfassungen als vollständig gelten können, obwohl sie inhaltlich keine Übereinstimmung aufweisen. Der deutsche Textteil enthält zusätzlich ein Bibelzitat und das Todesdatum nach der jüdischen Zeitrechnung. Inhaltlich entspricht die hebräische Eulogie nicht der deutschen: Beide thematisieren nach der jüdischen Tradition familiäre Verhältnisse, jedoch sprechen unterschiedliche Eigenschaften des Verstorbenen an. Die hebräischen Einleitungs- und Schlussformeln bilden einen Rahmen, der dem Gesamttext einen Charakter eines abgeschlossenen Ganzen verleiht. Das Psalmzitat im deutschen Textteil nimmt inhaltlich auf die Eulogie Bezug; der Platzierung nach übernimmt es die Rolle der fehlenden deutschsprachigen Schlussformel (Jarosz, 2013, S. 126).

${ }^{23}$ Beide angeführte Belege (mit den deutschen Übersetzungen aus dem Hebräischen) wurden der epigraphischen Datenbank http://steinheim-institut.de/cgi-bin/epidat entnommen [20.12.2015]. 
(4)

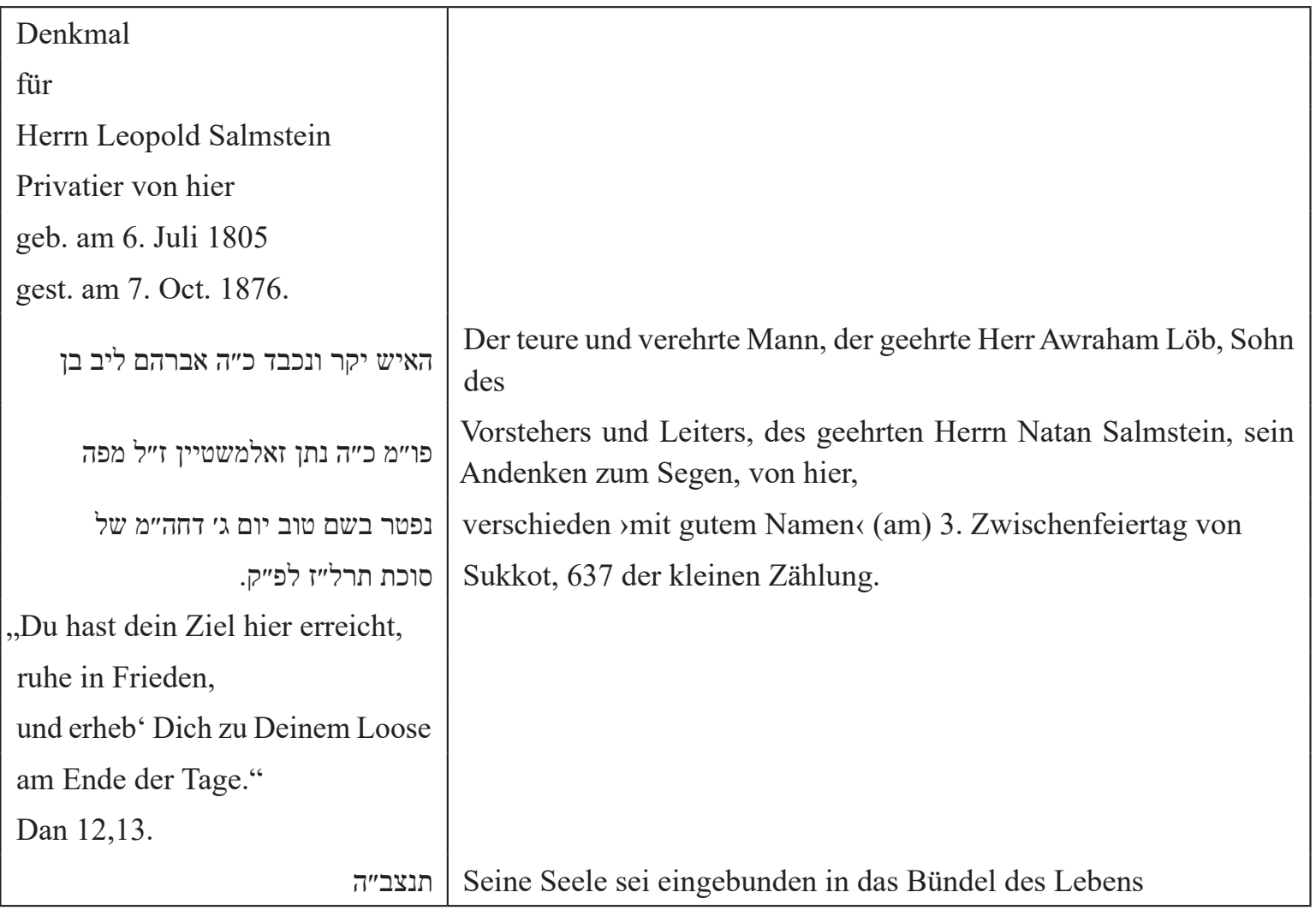

Nach der Analyse des Umfangs und der Platzierung der hebräischen Inschrift muss man die dominierende Funktion dem deutschen Text beimessen (Beispiel 4), der eine vollständige Inschrift mit den kanonischen Grundkomponenten bildet: Einleitungsformel, onomastisch-genealogische Angaben und Laudatio. Die Wünsche im angeführten Bibelzitat, das die Inschrift abschließt, ersetzen die fehlende Schlussformel. Eine ähnliche Funktion scheint der hebräische Segensspruch zu erfüllen. Während der letztere die komplementäre Beziehung in struktureller Hinsicht mit dem deutschen Text schafft, scheint die hebräische Eulogie inhaltlich teilweise redundant zu sein.

Beide Textbelege veranschaulichen, dass der Anteil des Deutschen und Hebräischen, sowie die Kombination der zweisprachigen Komponenten in den untersuchten Inschriften ein buntes Mosaik bilden. Sie variieren im Umfang sowie im Inhalt und gelten nicht als Übersetzungen. Der Gebrauch einer der Sprachen erfolgt in bestimmten Textsegmenten ohne übersichtliche Regeln oder Prinzipien. Die Wahl der Sprache sowie der Umfang der Textteile in beiden Sprachen hängen jeweils von den Auftraggebern ab. Eine umfassendere Untersuchung deutsch-hebräischer Grabinschriften (vgl. Jarosz, 2013) hat ergeben, dass man beiden Sprachen unterschiedliche Funktionen zuschreiben kann. Die in deutscher Sprache verfassten Textsequenzen haben einen eher offiziellen Charakter. Sie enthalten bürgerliche Namen, das Geburts- und Sterbedatum werden in der Regel nach christlichem Kalender angegeben. Manche Informationen wurden der 
hebräischen Inschrift - und damit dem innerjüdischen Kreis - vorbehalten. Sie geben gerade bei Frauen oftmals einen kleinen Einblick in das Privatleben.

In den hebräischen Eulogien werden nach der Tradition die familiären Verhältnisse, Rollen und Funktionen in der jüdischen Gemeinde erwähnt. Hier sind auch die genealogischen Angaben ausführlicher. Die hebräischen Passagen der Inschriften enthalten oft die häuslich-familiäre Ruf- oder Koseform des Namens, während in den deutschen Inschriften sich stets der korrekte offizielle Namen findet. Die alten ehrwürdigen Wendungen und Lobpreisungen werden auch in deutscher Sprache zum Ausdruck gebracht: sie werden meistens nicht wiederholt, sondern anders ausgedrückt. Der deutsche Text zeigt eine/n Bürger/in, der hebräische ein Familienmitglied und ein Mitglied der Gemeinde, wodurch die Verteilung der Rollen von Sprachen zum Ausdruck kommt. (Jarosz, 2013, S. 129)

Gewöhnlich vervollständigen sich inhaltlich und strukturell beide Textkomponenten und bilden damit eine komplexe Einheit, die das Leben und Sterben zwischen zwei Sprachen und zwei Kulturen, zwischen Tradition und Assimilation symbolisch ausdrückt. Die Texte wenden sich somit gleichzeitig an zwei Gruppen von Empfängern²4. Selten ist der Fall, dass beide Textfragmente als autonome Inschriften gelten können.

Die Zweisprachigkeit der jüdischen Grabinschriften zeugt einerseits von dem bewussten Umgang mit der deutschen Sprache und Kultur, andererseits ist sie ein Beweis für eine große Gebundenheit an die Tradition und Sprache der Ahnen. Der Gebrauch des Deutschen ist natürlich als signum temporis zu interpretieren, denn die früheren Inschriften (im 18. Jh. und früher) waren immer nur einsprachig. Die sprachliche Koexistenz auf den Grabplatten gilt auch als symbolischer Ausdruck des Zwiespalts zwischen Deutschtum und Judentum.

Die Funktion mehrsprachiger Textkomponenten in neueren Grabinschriften, deren Umfang viel bescheidener ist als der der jüdischen, wird am Beispiel von Originalbelegen von dänischen Friedhöfen erörtert (die Quellen werden jeweils in Klammern nach der angeführten Inschrift angegeben). Belege (5) und (6) sind kurze dänische Grabinschriften, in denen man eindeutig eine Kookurenz zweier Sprachen feststellen kann.

(5)

Prestur

Petur Andrew Petersen

*26.11.1927+8.3.2008

Du gav af hele dit hjerte

(Vestrekirkegård, København)
(6)

Danuta Rytter Kandel
LÆGE
9.10.1930 - 21.2.2009
MAMIE I BABCI
RODZINA

(Vestrekirkegård, København)

${ }^{24}$ Der festgestellte allmähliche Übergang von den einheitlichen monolingualen in der hebräischen Sprache verfassten Texte bis zum Schwund des Hebräischen aus der Fläche der Grabplatten hängt mit der Assimilation der Juden zusammen, die übrigens als eine unmittelbare Ursache für das Verfassen der Inschriften in zwei Sprachen im Laufe des 19. Jahrhunderts und der ersten Hälfte des 20. Jahrhunderts zu betrachten ist (Jarosz, 2013, S. 129). 
Das Wort prestur, das als Berufsbezeichnung in der Muttersprache des Verstorbenen die erste Inschrift einleitet, ist die färöische Bezeichnung für Priester (dän. prcest). Den Text schließt eine kurze Laudatio in dänischer Sprache: Du gav af hele dit hjerte (dt. ,Du hast dein ganzes Herz abgegeben') ab. Umgekehrte Verhältnisse im Sprachgebrauch beobachtet man in einer anderen Inschrift, die von dem gleichen Friedhof stammt und ein Jahr jünger als der erste Textbeleg ist. Die Berufsbezeichnung wurde in dänischer Sprache ausgedrückt: loege (dt. ,Arzt/Ärztin'), der dedikatorische Teil, in dem die Stifter zum Wort kommen und der die Rolle der Schlussformel übernimmt, wurde in der Muttersprache der Verstorbenen formuliert Mamie i Babci-Rodzina (dt. ,Der Mutti und Omi - Familie'). Die fremdsprachigen (d. h. färöischen und polnischen) Elemente erfüllen trotz ihrer Kürze eine wichtige Aufgabe. Durch den Gebrauch der Muttersprache der Verstorbenen wird ihre ethnische und kulturelle $\mathrm{Zu}$ gehörigkeit mitgeteilt und hervorgehoben. Die Muttersprache gilt im Ausland als Teil der eigenen Identität. Grabplatten bzw. Grabsteine bieten eine einmalige Gelegenheit, die Verstorbenen der Öffentlichkeit mit Gebrauch sprachlicher oder visueller Mittel vorzustellen. Beide Textbelege wenden sich an zweisprachige Rezipienten, bei denen sich das bilinguale Informationspotenzial entfalten kann. Im Beispieltext (6) leistet der polnische Teiltext zusätzlich einen Beitrag zur starken Emotionalisierung der Inschrift. Dies wurde durch die kontrastive Gegenüberstellung des beruflichen Bereichs (in dem die Berufsbezeichnung in der offiziellen Landessprache ausgedrückt wurde) und des privaten Bereichs (familiäre Verhältnisse wurden in der Muttersprache als Indikatoren der Emotionalität formuliert) erreicht.

Grabinschriften dienen der Memoria und funktionieren als eine lokale Landesgeschichte und Stadtchronik, in der jeder Bürger einen Platz für sich haben kann (vgl. Jarosz, 2017, S. 73, 246).

Eine andere Funktion ist wohl den fremdsprachlichen Inschriften auf dem Grabmal von Rasmus Rask zuzuschreiben. Der bekannte dänische Sprachforscher (1787-1832), Indogermanist und Polyglott lebte in Kopenhagen, wo er auch beerdigt wurde (Assistens Kirkegård, København). Seinen Grabstein zieren Inschriften in vier Sprachen, wodurch sie nicht nur durch den Textumfang, sondern auch durch ihre äußere Erscheinung als ein Blickfang gelten können: Die Stifter ließen Inschriften auf Dänisch, Arabisch, Isländisch, Sanskrit und in Runenzeichen in Stein meißeln (Abb. 1):

Dieses bunte Mosaik sorgt für einen ungewöhnlichen visuellen Effekt und würdigt den Wissenschaftler mit der Materie, der er sein ganzes Leben gewidmet hat - den Fremdsprachen. Der Gebrauch von Fremdsprachen dient zur Charakterisierung des Verstorbenen, zum Ausdruck seiner beruflichen Identität, steht also mit anderen sprachlich-visuellen Elementen, die gewöhnlich auf Grabplatten zu finden sind, im Einklang.

Die Koexistenz unterschiedlicher Alphabete und Schriftarten ist somit in ihrer Funktion mit dem Wappen einer Zunft oder eines Handwerkerverbandes auf einer Grabplatte vergleichbar, die als Ausdruck der Identität und Zugehörigkeit der Verstorbenen auf Grabsteine gesetzt werden. 


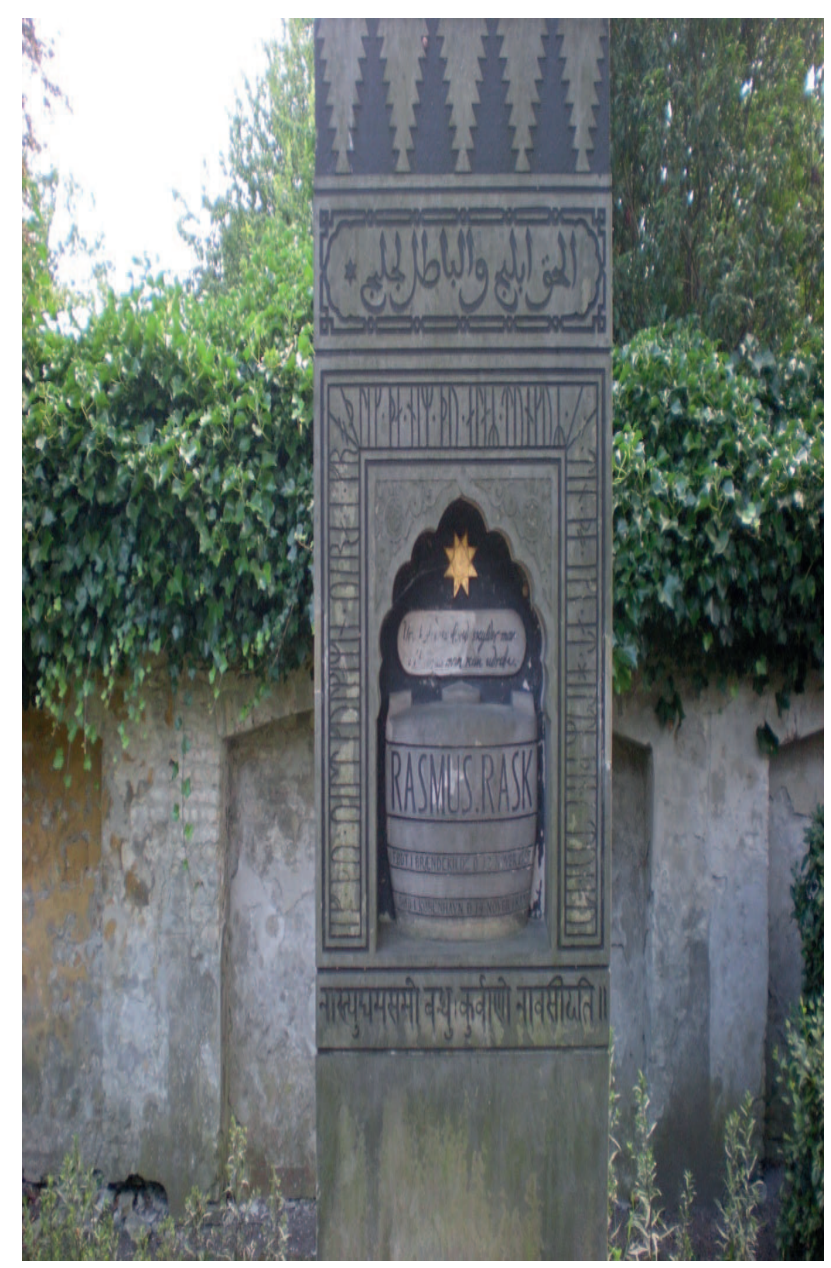

Abb. 1: Mehrsprachige Inschrift auf dem Grabstein von Rasmus Rask auf dem Assistens Kirkegård in Kopenhagen (J. Jarosz)

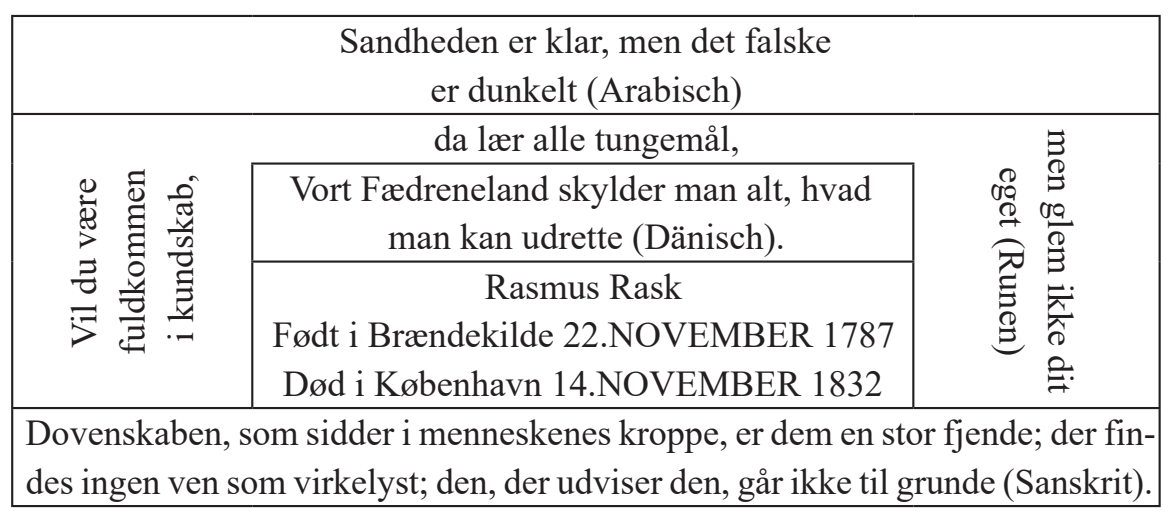

Abb. 2: Schematische Darstellung der Teilinschriften (auf Dänisch) auf dem Grabstein von Rasmus Rask

Mit der Verwendung einer Muttersprache oder Fremdsprache in Grabinschriften wird die ethnische oder berufliche Identität zum Ausdruck gebracht. Die Präsenz von Fremdsprachen dient somit zur Identifizierung und Individualisierung. Hier überlappen sich ihre Funktionen mit der Funktion der Textsorte. 


\section{Schlussbemerkungen}

Die in dem vorliegenden Beitrag unternommene Untersuchung der Funktion der Zweisprachigkeit in Textsorten ergab einige Beobachtungen, die in Form einer Typologie in der folgenden Tabelle zusammengestellt werden:

Tab. 1: Formen und Funktionen von Mehrsprachigkeit in Textsorten

\begin{tabular}{|c|c|c|c|}
\hline \multicolumn{2}{|c|}{$\begin{array}{l}\text { Zweisprachigkeit } \\
\text { und ihre Formen }\end{array}$} & $\begin{array}{l}\text { Funktion der Mehrsprachigkeit } \\
\text { im Text }\end{array}$ & Namen von Textsorten \\
\hline \multirow{2}{*}{\multicolumn{2}{|c|}{ textexterne Zweisprachigkeit }} & Keine zusätzliche Funktion & $\begin{array}{l}\text { mehrsprachige Broschüren mit } \\
\text { Gebrauchsanweisungen, Bedie- } \\
\text { nungsanleitungen, Informationen } \\
\text { auf Verpackungen }\end{array}$ \\
\hline & & $\begin{array}{l}\text { zusätzliche Funktion beim Über- } \\
\text { setzungsvergleich - Einblick in } \\
\text { die Technik des Übersetzens }\end{array}$ & zweisprachige Gedichtbände \\
\hline \multirow{3}{*}{$\begin{array}{l}\text { Textinterne } \\
\text { Zweisprachig- } \\
\text { keit }\end{array}$} & obligatorisch & $\begin{array}{l}\text { konstituiert den Text und seine } \\
\text { Grundfunktion }\end{array}$ & $\begin{array}{l}\text { mehrsprachige Wörterbücher } \\
\text { aller Art, Sprachführer, Sprach- } \\
\text { lehrbücher }\end{array}$ \\
\hline & \multirow{2}{*}{ fakultativ } & unterstützt die Grundfunktion & Werbetexte, Grabinschriften \\
\hline & & Keine zusätzliche Funktion & wissenschaftliche Sammelbände \\
\hline
\end{tabular}

Neben einigen Beobachtungen, die an der Grenze der Banalität balancieren, sind einige, die einiger Ergänzungen oder Untersuchungen an anderen Textsorten bzw. an einem umfangreicheren Korpus bedürfen. Als Mehrwert dieser Untersuchung gilt die Konstatierung, die in zwei unterschiedlichen Textsorten eine Bestätigung fand, dass die Zweisprachigkeit als ein zusätzliches Mittel zur Unterstützung der dominierenden Textfunktion verwendet werden kann.

\section{Literatur}

Adamzik, K. (1995). Textsorten - Texttypologie. Eine kommentierte Bibliographie. Münster: Nodus. Adamzik, K. (2016). Kulturwissenschaftliche Orientierung in der Textlinguistik. In L. Jäger \& W. Holly \& P. Krapp \& S. Weber (Hrsg.), Sprache - Kultur - Kommunikation. Ein internationales Handbuch zu Linguistik als Kulturwissenschaft (S. 818-828). Berlin-New York: de Gruyter.

Bohmann, S. (1996). Englische Elemente im Gegenwartsdeutsch der Werbebranche. Marburg: C.H.BECK. Brinker, K. (1983). Textfunktionen. Ansätze zu ihrer Beschreibung. Zeitschrift für Germanistische Linguistik, 11, 127-148.

Brinker, K. (2010). Textlinguistik. Tübingen: Erich Schmidt.

Dedecius, K. (1982). Sto wierszy polskich w wyborze i ttumaczeniach Karla Dedeciusa. Hundert polnische Gedichte, ausgewählt und übertragen von Karl Dedecius. Kraków: Wydawnictwo Literackie.

Eckkrammer, E.M. \& Eder, H.M. (2000). (Cyber)Diskurs zwischen Konvention und Revolution. Eine multilinguale textlinguistische Analyse von Gebrauchstextsorten im realen und virtuellen Raum. Frankfurt a. M. et al.: Peter Lang. 
Elsen, H. (2008). Englische Elemente in der Kosmetikwerbung. Deutsche und französische Anzeigen im Vergleich. In B. Dumiche \& H. Klöden (Hrsg.), Werbung und Werbesprache: eine Analyse im interdisziplinären Kontext (S. 87-121). Wilhelmsfeld: Egert.

Engel, U. et al. (2000). Deutsch-polnische kontrastive Grammatik, Bd. 1-2. Warszawa: Wydawnictwo Naukowe PWN.

Ermert, K. (1979). Briefsorten: Untersuchungen zu Theorie und Empirie der Textklassifikation. Tübingen: Max Niemeyer.

Fandrych, Ch. \& Thurmair, M. (2011). Textsorten im Deutschen. Linguistische Analysen aus sprachdidaktischer Sicht. Tübingen: Stauffenburg.

Fix, U. (2011b). Was macht eine kulturspezifisch orientierte Textlinguistik aus? Überlegungen und Beispiele. In M.L. Kotin \& E.G. Kotorova (Hrsg.), Die Sprache in Aktion (S. 145-155). Heidelberg: Winter.

Furthmann, K. (2006). Die Sterne lügen nicht: Eine linguistische Analyse der Textsorte Pressehoroskop. Göttingen: V und R Unipress.

Göpferich, S. (1995). Textsorten in Naturwissenschaften und Technik. Pragmatische Typologie - Kontrastierung - Translation. Tübingen: Narr.

Guthke, K.S. (2006). Sprechende Steine: eine Kulturgeschichte der Grabschrift. Göttingen: Wallstein-Verl.

Hartmann, P. (1971). Texte als linguistisches Objekt. In W.-D. Stempel (Hrsg.), Beiträge zur Textlinguistik (S. 9-29). München: Fink.

Hartmann, R.R.K. (1980). Contrastive Textology. Heidelberg: Groos.

Hauser, S. \& Kleinberger, U. \& Roth, K.S. (Hrsg.). (2014). Musterwandel - Sortenwandel. Aktuelle Tendenzen der diachronen Text(sorten)linguistik. Bern u. a.: Peter Lang.

Heinemann, W. (2000). Textsorte - Textmuster - Texttyp. In K. Brinker \& G. Antos \& W. Heinemann \& S.F. Sager (Hrsg.), Text- und Gesprächslinguistik. Handbücher zur Sprach- und Kommunikationswissenschaft. Bd. 16. 1. Halbband. Textlinguistik (S. 507-523). Berlin-New York: de Gruyter.

Heinemann, W. \& Viehweger, D. (1991). Textlinguistik. Eine Einführung. Tübingen: Max Niemeyer.

Hofeneder, P. (2013). Die mehrsprachige Ukraine. Übersetzungspolitik in der Sowjetunion von 1917 bis 1991. Münster-Wien: LIT Verlag.

Hoffmann, H.-J. (1981). Psychologie der Werbekommunikation. 2., neu bearbeitete Auflage. Berlin-New York: de Gruyter.

Hosselmann, B. (2001). Todesanzeigen als memento mori. Eine empirische Untersuchung von Todesanzeigen der Gegenwart. Altenberge: Oros.

Jarosz, J. (2011). Z dziejów paremiografii duńskiej. Część 1: Wiek XVI. Orbis Linguarum, 37, 471-486.

Jarosz, J. (2013). Leben und Tod zwischen zwei Sprachen und Kulturen. Zur Problematik der zweisprachigen jüdischen Grabinschriften. Studia Linguistica, 32, 117-131.

Jarosz, J. (2015). Grabinschriften im Textsortennetz. In D. Kaczmarek \& M. Michoń \& J. Makowski \& Z. Weigt (Hrsg.), Text-Wesen. Theorien und Analysen (S. 23-34). Łódź: Wydawnictwo Uniwersytetu Łódzkiego.

Jarosz, J. (2017). Grabinschrift - eine Textsorte im Wandel. Eine diachrone Studie am deutschen epigrafischen Material 1780-2015. Wrocław-Dresden: Atut-Neisse Verlag.

Kupper, S. (2007). Anglizismen in deutschen Werbeanzeigen. Eine empirische Studie zur stilistischen und ökonomischen Motivation von Anglizismen. Frankfurt am Main: Peter Lang.

Luginbühl, M. (2014). Medienkultur und Medienlinguistik. Komparative Textsortengeschichte(n) der amerikanischen "CBS Evening News» und der Schweizer «Tagesschau». Bern u. a.: de Gruyter.

Lux, F. (1981). Text, Situation, Textsorte. Probleme der Textsortenanalyse, dargestellt am Beispiel der britischen Registerlinguistik. Mit einem Ausblick auf eine adäquate Textsortentheorie. Tübingen: Narr.

Łuszczyk, S. \& Szulc, A. \& Wawrzyniak, Z. (1988). Gramatyka języka niemieckiego. Warszawa: WSiP.

Mickiewicz, A. (1979). Lyrik. Prosa. Polnisch und deutsch. Leipzig: Reclam. 
Józef Jarosz

Pöckl, W. (2010). Eurotextologie. In U. Hinrichs (Hrsg.), Handbuch der Eurolinguistik (S. 729-738). Wiesbaden: Harrassowitz Verlag.

Rinnekangas, A. (2013). Das persönliche Glück auf dem unpersönlichen Markt. Deutsche und finnische Kontaktanzeigen im 20. Jahrhundert. Eine kontrastive und diachrone Textsortenuntersuchung. Åbo: Akademi.

Rolf, E. (1993). Die Funktionen der Gebrauchstextsorten. Berlin: de Gruyter.

Schenker, W. (1977). Plädoyer für eine Sprachgeschichte als Textsortengeschichte. Dargestellt am Paradigma von Telefon, Radio, Fernsehen. Deutsche Sprache, 5, 141-148.

Schmitt, R. (1986). Auswahlbibliographie zur Kommunikationstypologie aus sprachwissenschaftlicher Perspektive. In W. Kallmeyer (Hrsg.), Kommunikationstypologie. Handlungsmuster, Textsorten und Situationstypen. Jahrbuch 1985 des Instituts für deutsche Sprache (S. 337-384). Düsseldorf: Schwann.

Schütte, D. (1996). Das schöne Fremde. Anglo-amerikanische Einflüsse auf die Sprache der deutschen Zeitschriftenwerbung. Opladen: Westdeutscher Verlag.

Sommerfeldt, K.-E. (2002). Zur Gestaltung von Kontaktanzeigen in verschiedenen Printmedien. In I. Pohl (Hrsg.), Semantische Aspekte öffentlicher Kommunikation (S. 329-350). Frankfurt am Main u. a.: Peter Lang.

Spillner, B. (1981). Textsorten im Sprachvergleich. Ansätze zu einer kontrastiven Textologie. In W. Kühlwein \& G. Thome \& W. Wilss (Hrsg.), Kontrastive Linguistik und Übersetzungswissenschaft. Akten des Internationalen Kolloquiums (Trier/Saarbrücken 25.-30.9.1978) (S. 239-250). München: Fink.

Spillner, B. (1983). Zur kontrastiven Analyse von Fachtexten - am Beispiel der Syntax von Wetterberichten. LiLi. Zeitschrift für Literaturwissenschaft und Linguistik, 13, H. 51/52, Fachsprache und Fachliteratur, 110-123.

Spillner, B. (2002). Fachtexte im interkulturellen Vergleich. Kontrastive Pragmatik deutscher, finnischer und französischer Wirtschaftstexte. In A. Nuopponen \& T. Harakka \& R. Tatje (Hrsg.), Interkulturelle Wirtschaftskommunikation. Forschungsobjekte und Methoden (S. 144-164). Vaasa: Universität Vaasa.

Steger, H. (1998). Sprachgeschichte als Geschichte der Textsorten, Kommunikationsbereiche und Semantiktypen. In W. Besch u. a. (Hrsg.), Sprachgeschichte. Ein Handbuch zur Geschichte der deutschen Sprache und ihrer Erforschung (S. 284-300). Berlin-New York: de Gruyter.

Szczęk, J. (2015). Absageschreiben auf Bewerbungen. Eine pragmalinguistische Studie. Berlin: Frank \& Timme.

Szurawitzki, M. (2011). Der thematische Einstieg. Eine diachrone und kontrastive Studie auf der Basis deutscher und finnischer linguistischer Zeitschriftenartikel. Frankfurt am Main et al.: Peter Lang.

Weidenbusch, W. (2006a). Historische Textsortenlinguistik: Exemplarische Fallstudien. In G. Ernst \& M.D. Gleßgen \& Ch. Schmitt \& W. Schweickard (Hrsg.), Romanische Sprachgeschichte/Histoire des langues romanes (S. 2283-2292). Berlin-New York: de Gruyter.

Weidenbusch, W. (2006b). Historische Textsortenlinguistik: Theorie und Aufgabenbereiche. In G. Ernst \& M.-D. Gleßgen \& Ch. Schmitt \& W. Schweickard (Hrsg.), Romanische Sprachgeschichte/Histoire des langues romanes (S. 2278-2283). Berlin-New York: de Gruyter.

Weigt, Z. (2013). Die Textsorte Anzeige in der Lodzer Zeitung. In M.K. Lasatowicz \& A. Rudolph (Hrsg.), Corpora und Canones. Schlesien und andere Räume in Sprache, Literatur und Wissenschaft (S. 351363). Berlin: trafo.

Werlich, E. (1975). Typologie der Texte. Entwurf eines textlinguistischen Modells zur Grundlegung einer Textgrammatik. Heidelberg: Quelle \& Meyer.

Ziegler, E. (2013). Metropolenzeichen: Visuelle Mehrsprachigkeit in der Metropole Ruhr. Zeitschrift für Germanistische Linguistik, 41 (2), 299-301.

Zillig, W. (1982). Textsorte „, Rezension “. In K. Detering \& J. Schmidt-Radefeldt \& W. Sucharowski (Hrsg.), Sprache erkennen und verstehen (S. 197-208). Tübingen: Max Niemeyer. 
Ziting, Z. (2013). Wandel der Textsorte, Wandel der Kultur: Kontrastive Analyse und diachronischer Vergleich deutscher und chinesischer wissenschaftlicher Rezensionen. Lili. Zeitschrift für Literaturwissenschaft und Linguistik, 43, 142-162.

\section{Internetquellen}

Androutsopoulos, J.K. et al. (2004). Sprachwahl im Werbeslogan. Zeitliche Entwicklung und branchenspezifische Verteilung englischer Slogans in der Datenbank von slogans.de. Hannover. Abgerufen von http://www.mediensprache.net/networx/networx-41.pdf

Fix, U. (2011a). Was ist kulturspezifisch an Texten? Argumente für eine kulturwissenschaftlich orientierte Textsortenforschung. Abgerufen von http://home.uni-leipzig.de/fix/Was $\% 20$ ist $\% 20$ kulturspezifisch $\% 20$ an\%20Texten.pdf 